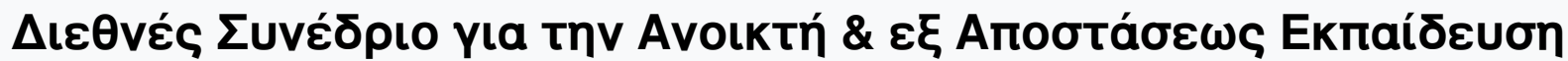

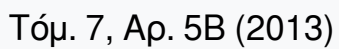

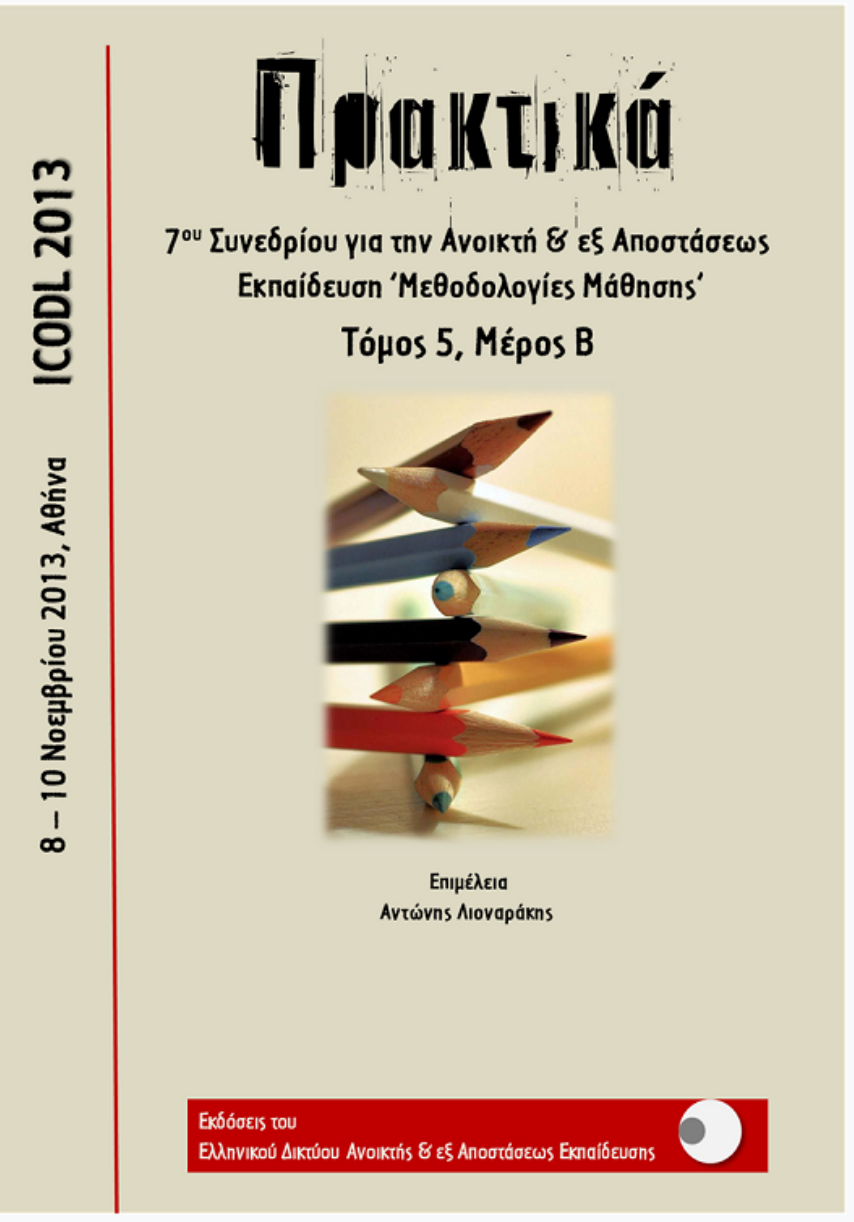

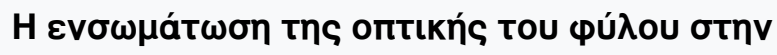

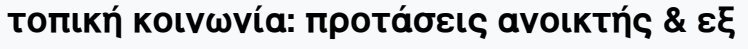

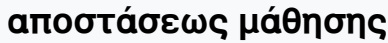

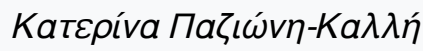

doi: $10.12681 /$ icodl. 559 


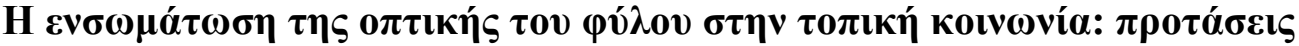

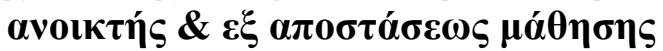

\section{Gender mainstreaming in local communities: open \& distance learning suggestions}

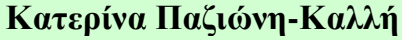

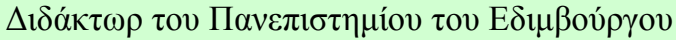

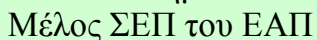 \\ kalipazioni@gmail.com
}

\begin{abstract}
Good local governance cannot work if due attention is not given to gender equality and women's empowerment. This is why sustained efforts should be made to incorporate gender both internally and in its work with open \& lifelong learning initiatives. This paper highlights the gender dimension and makes an effort to incorporate it into ongoing training activities and open learning suggestions in order to further support gender policies in local communities. Despite efforts on national and international level, many local government institutions are neither adequately equipped to provide specific training on gender, nor have they been able to integrate the gender dimension into their day-to-day practices, not to even mention complete lack of gender sensitiveness and enhance of relevant training for that matter. Therefore, a sustained policy for gender equality should include carefully designed training material, a provision for higher education institutions to be included as partners in mainstreaming gender education of local authorities for capacity-building regarding gender concerns.
\end{abstract}

Keywords: gender analysis, gender equality, community, open \& distance learning

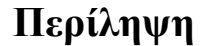

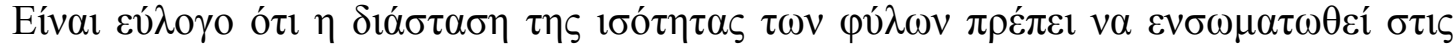

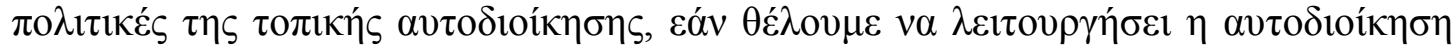

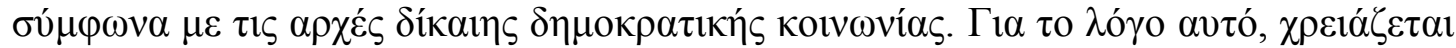

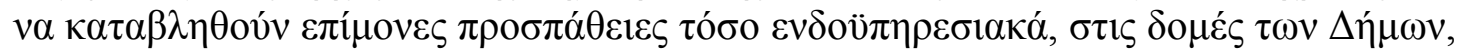

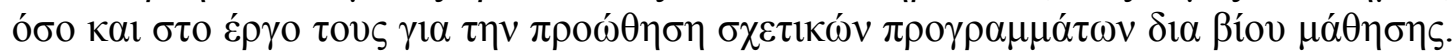

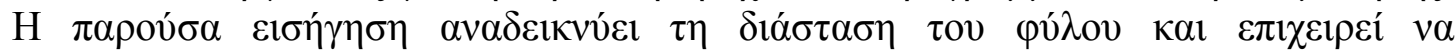

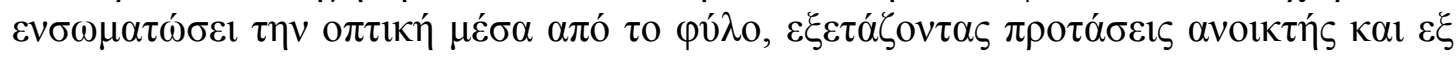

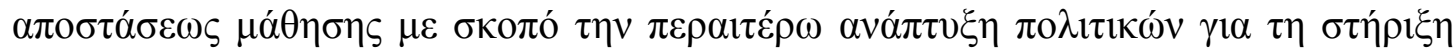

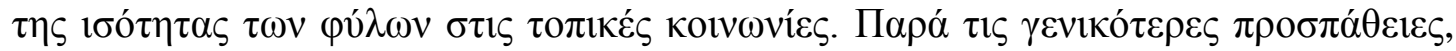

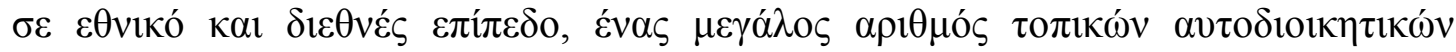

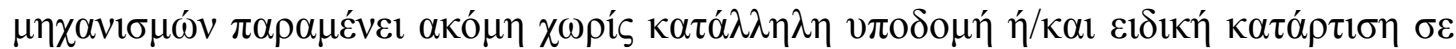

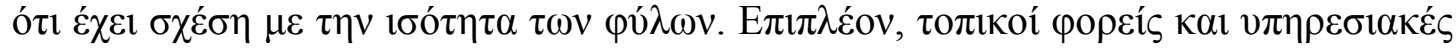

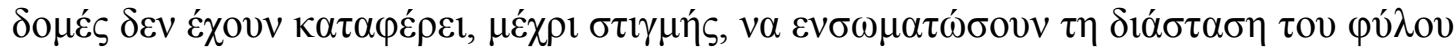

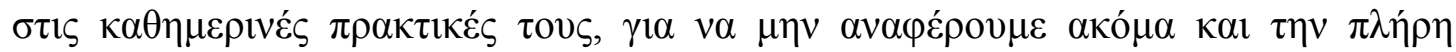

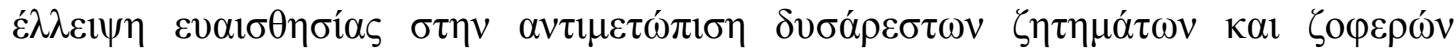




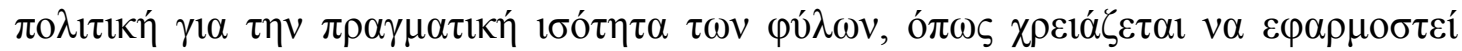

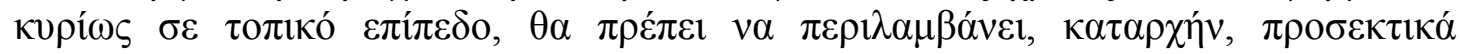

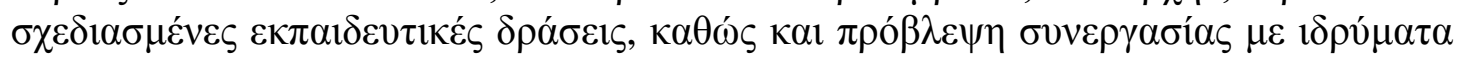

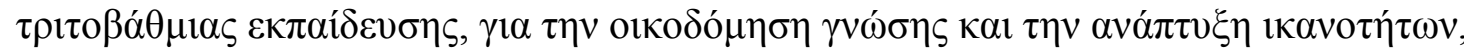

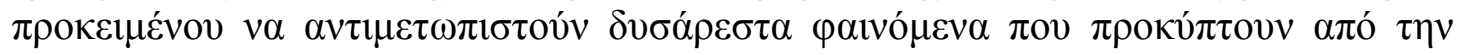

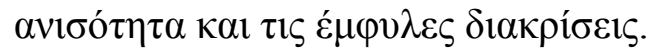

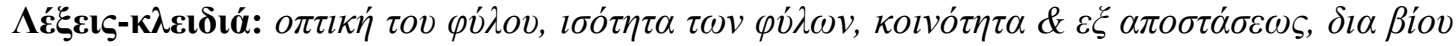
$\mu \alpha \dot{\theta} \theta \eta \sigma \eta$

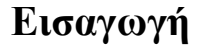

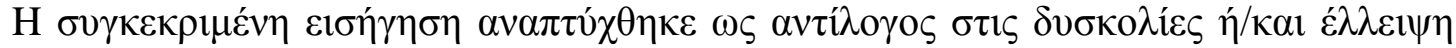

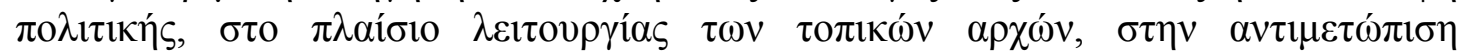

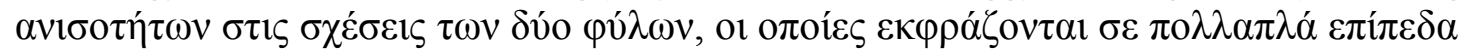

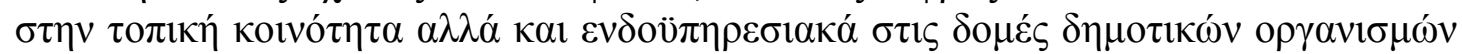

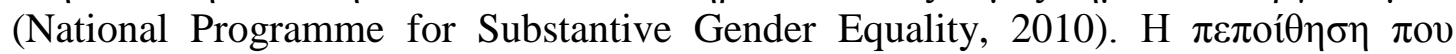

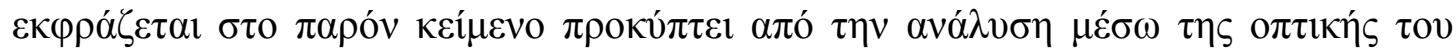

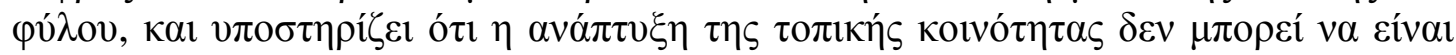

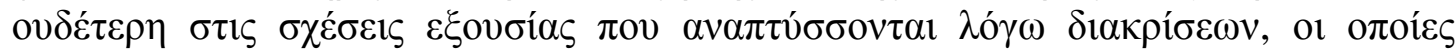

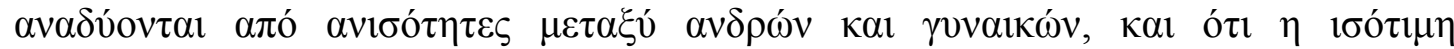

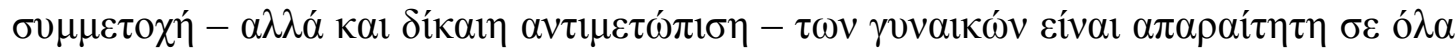

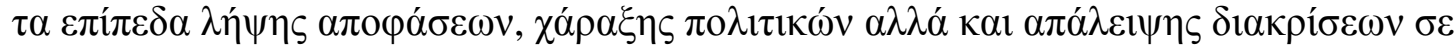

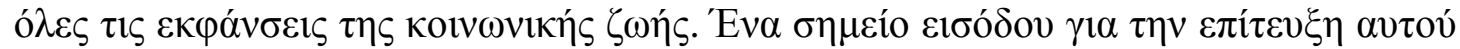

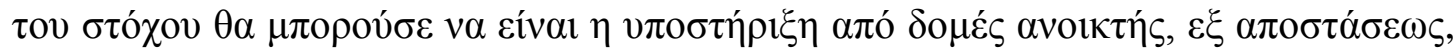

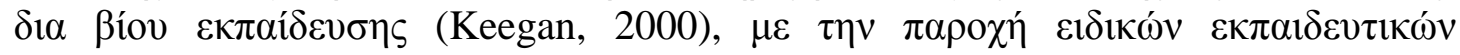

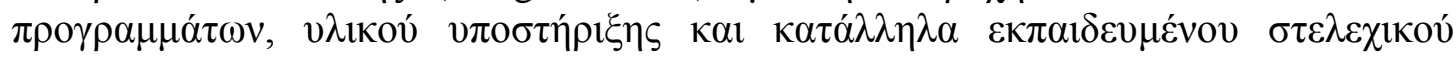

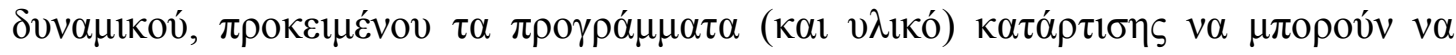

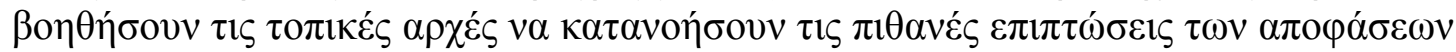

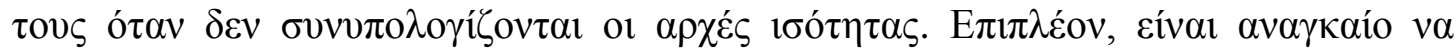

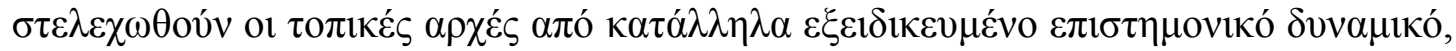

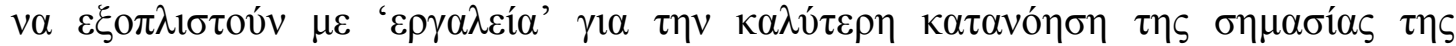

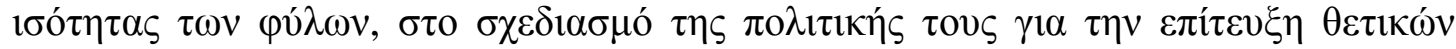

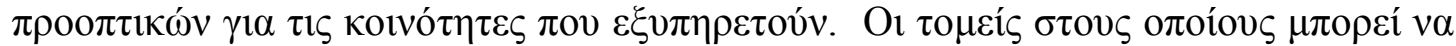

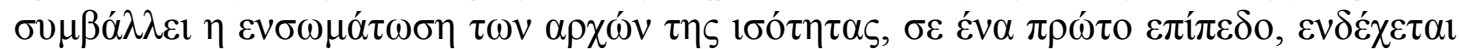
$v \alpha$ Eíval:

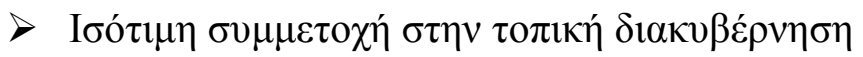

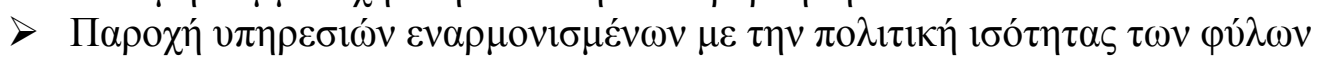

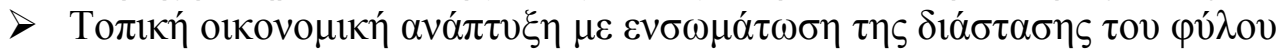

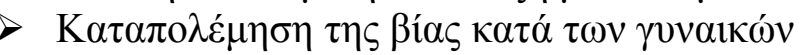

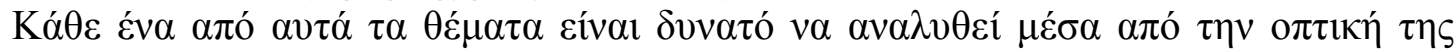

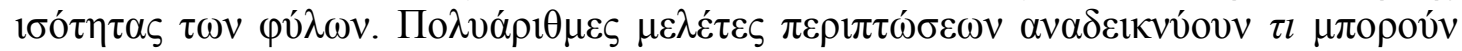

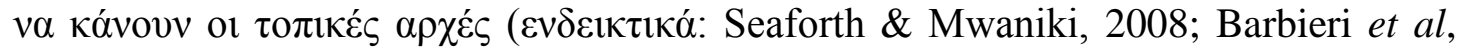

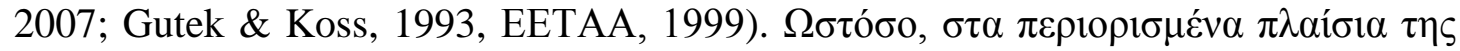

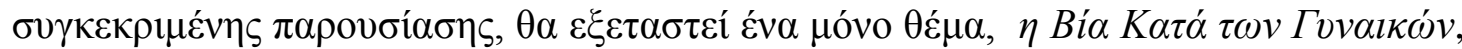

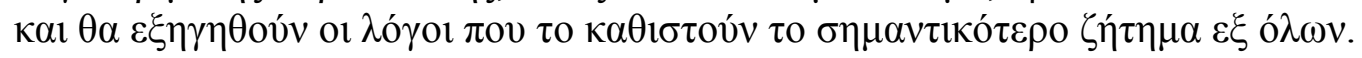




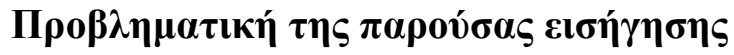

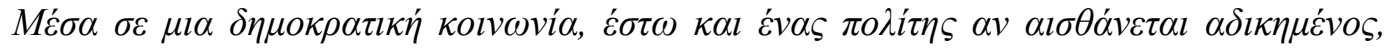
$\tau o ́ \tau \varepsilon \alpha v \tau \dot{\eta} \eta \delta \eta \mu \kappa \rho \alpha \tau i ́ \alpha \delta \varepsilon v \beta \alpha \delta i \zeta \varepsilon l \sigma \omega \sigma \tau \dot{\alpha}$

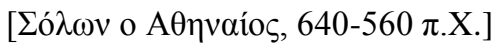

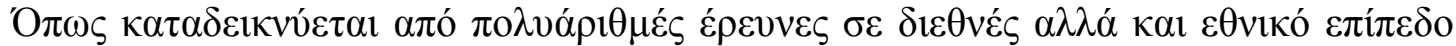

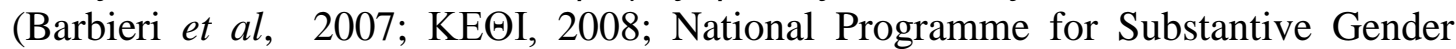

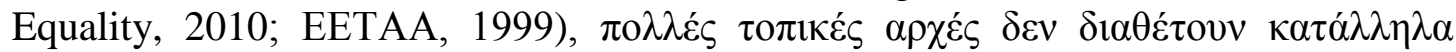

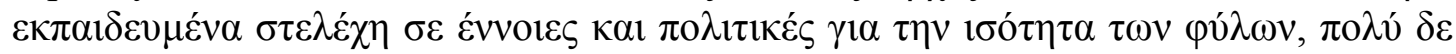

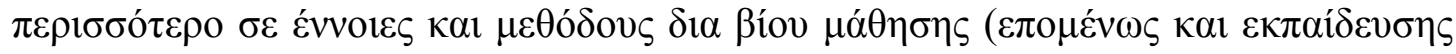

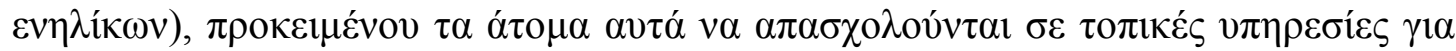

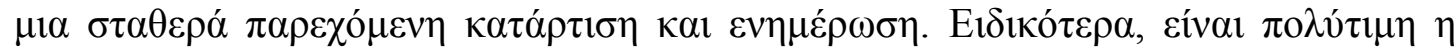

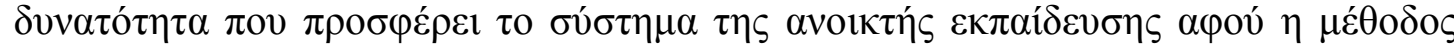

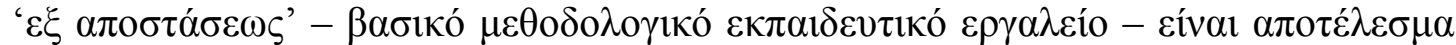

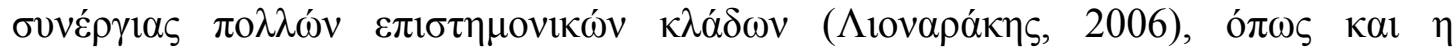

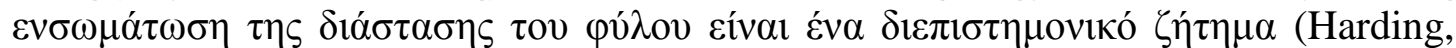

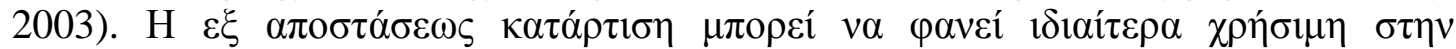

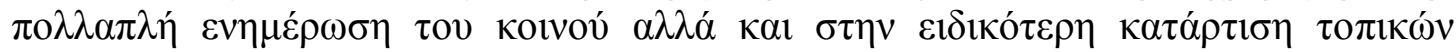

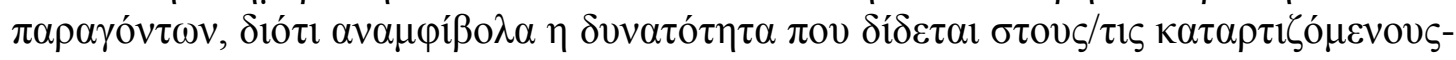

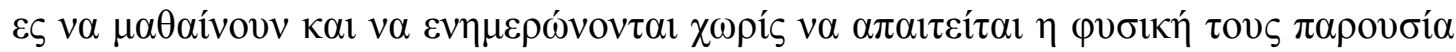

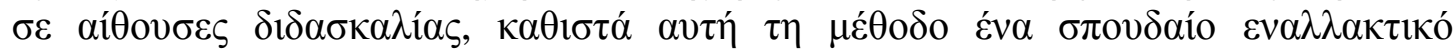

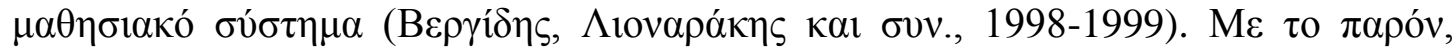

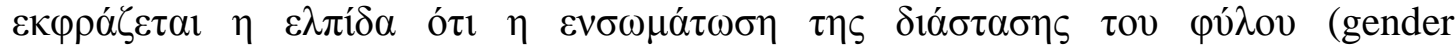

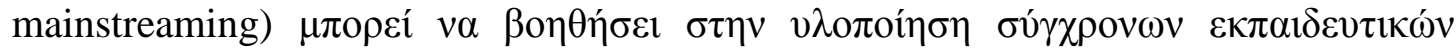

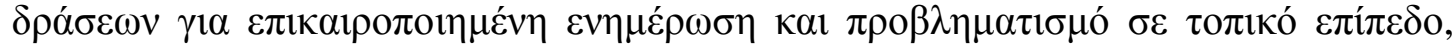

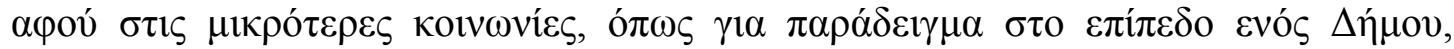

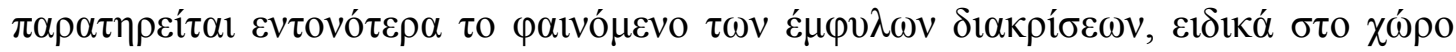

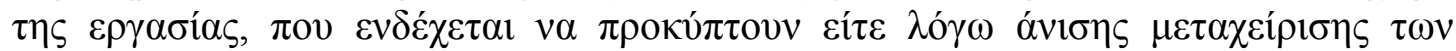

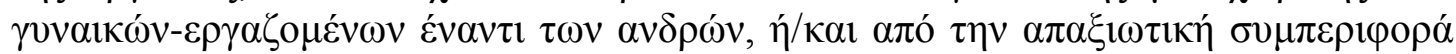
$\tau \omega v \delta \varepsilon v ́ \tau \varepsilon \rho \omega v \pi \rho \circ \varsigma \tau \iota \varsigma \pi \rho \omega ́ \tau \varepsilon \varsigma$ (Seaforth \& Mwaniki, 2008).

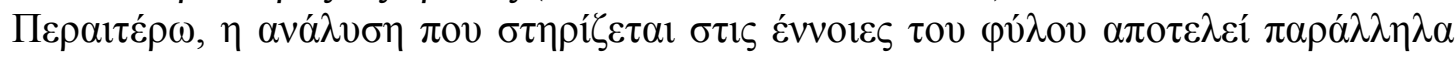

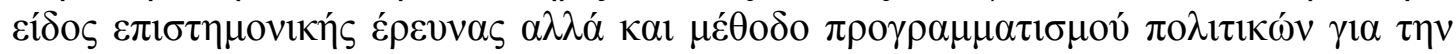

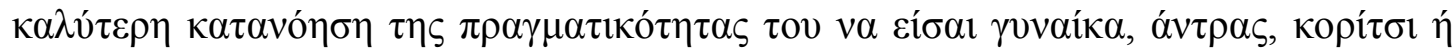

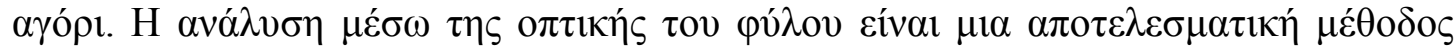

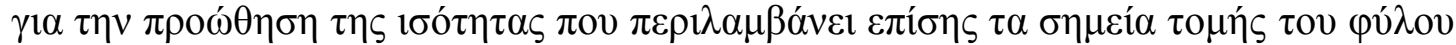

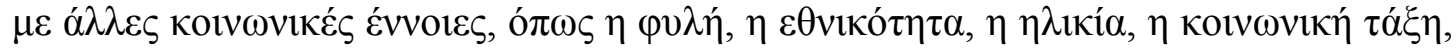
$\eta \sigma \varepsilon \xi o v \alpha \lambda \iota \kappa o ́ \tau \eta \tau \alpha, \eta \gamma \lambda \omega ́ \sigma \sigma \alpha, \eta \theta \rho \eta \sigma \kappa \varepsilon i ́ \alpha, \kappa \lambda \pi$ (Merrill, 2005; Tett, 2002; Thompson,

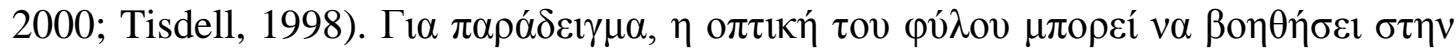

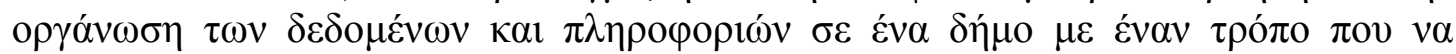

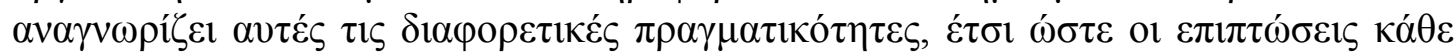

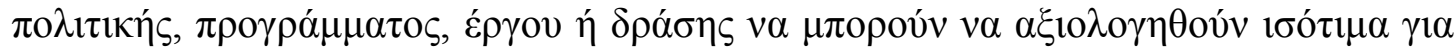

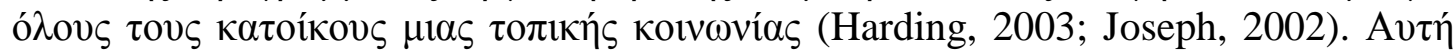

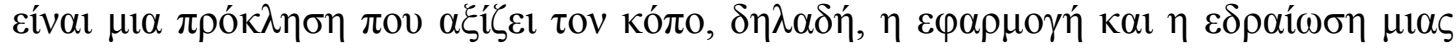

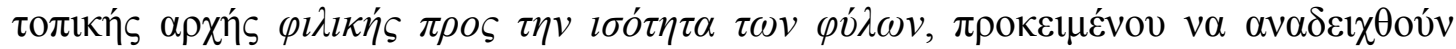

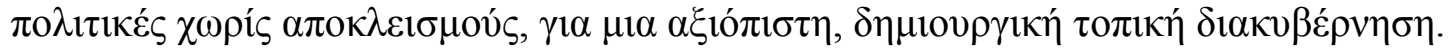

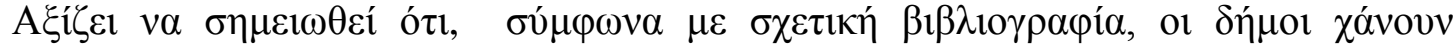

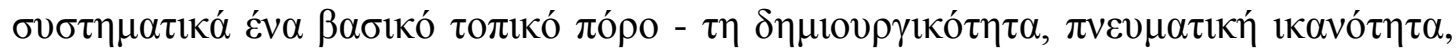

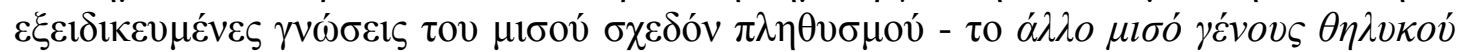




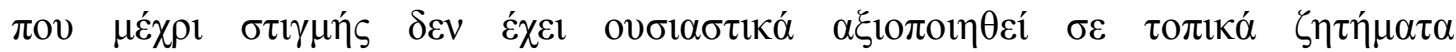

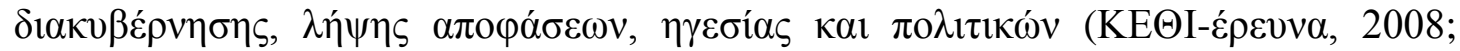

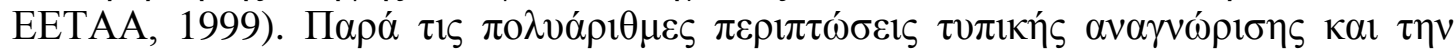

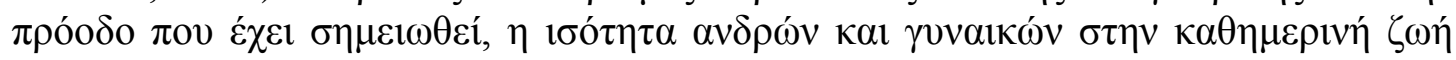

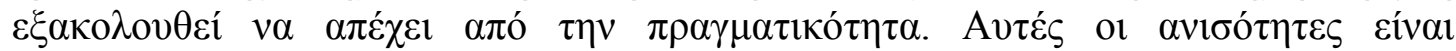

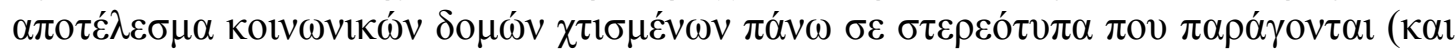

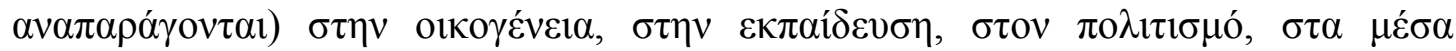

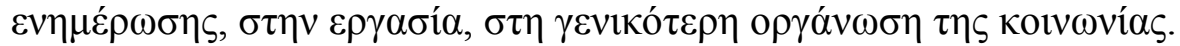

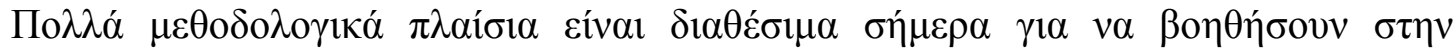

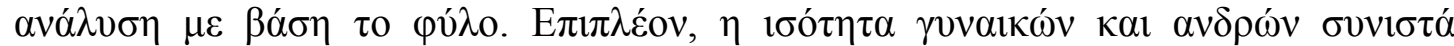

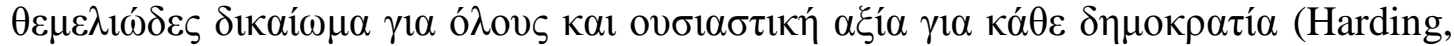

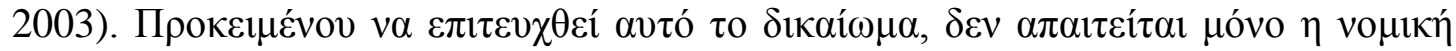

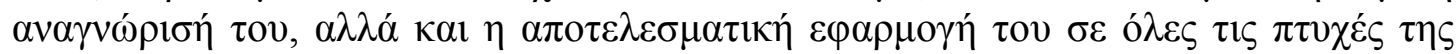

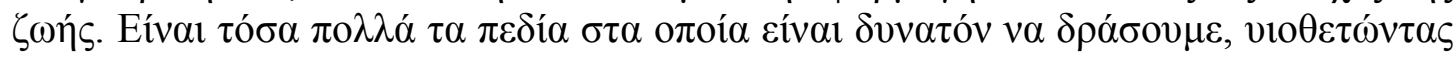

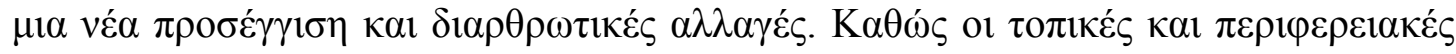

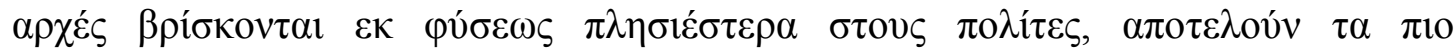

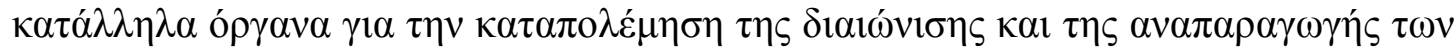

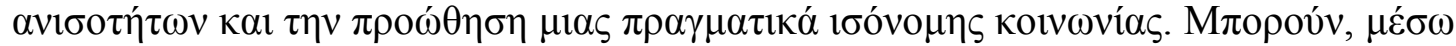

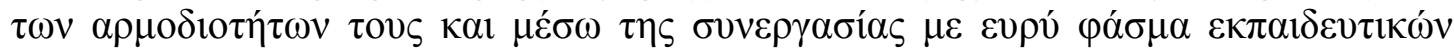

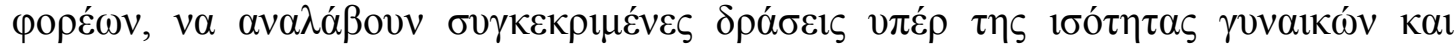

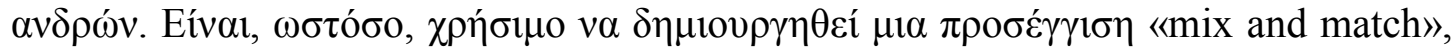

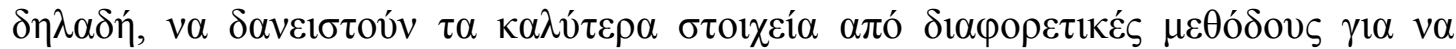

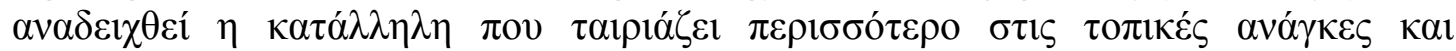

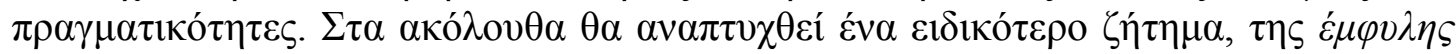

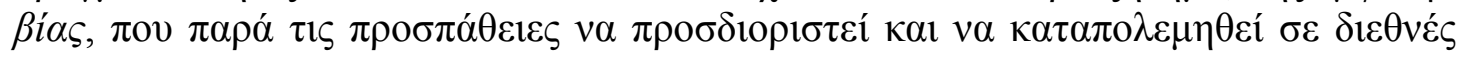

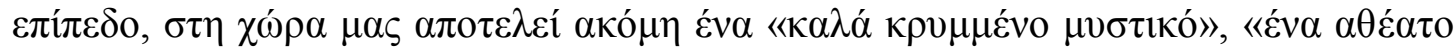

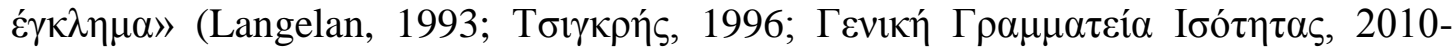

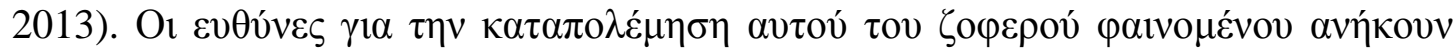

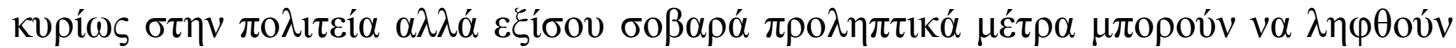

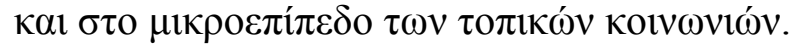

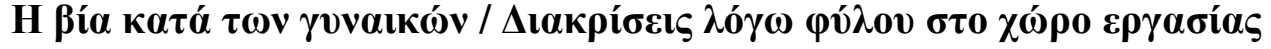

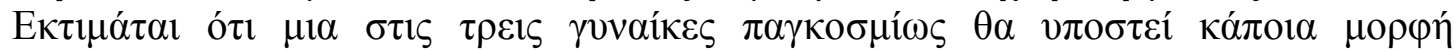

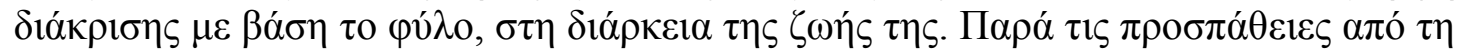

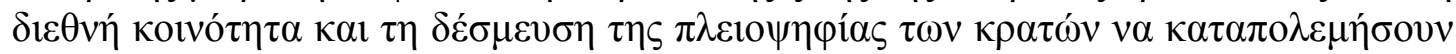

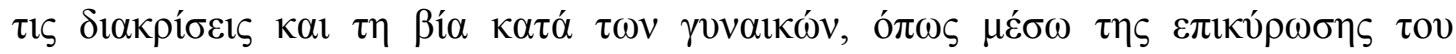

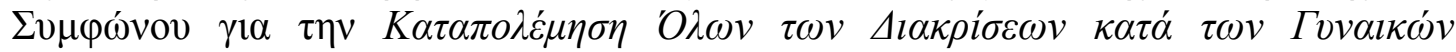

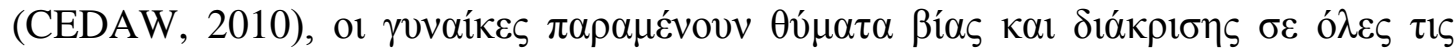

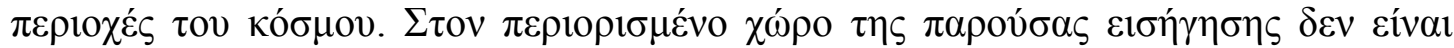

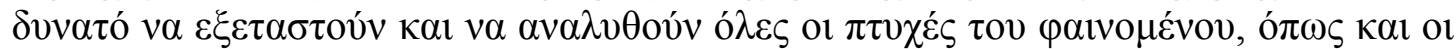

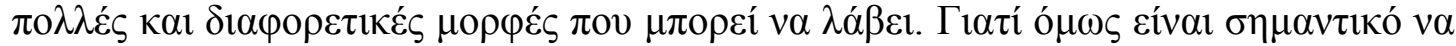

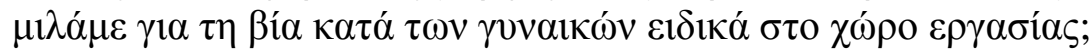

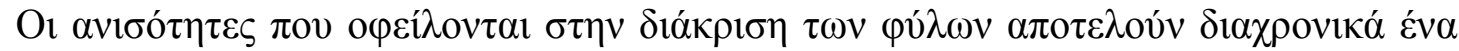

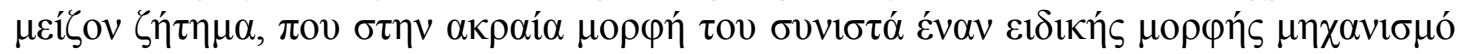

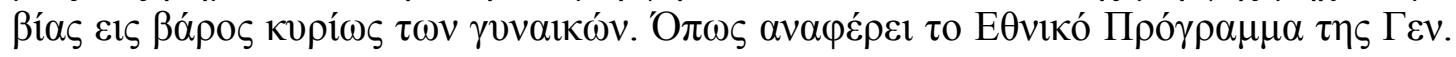

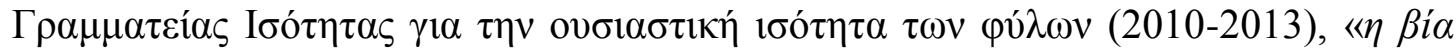

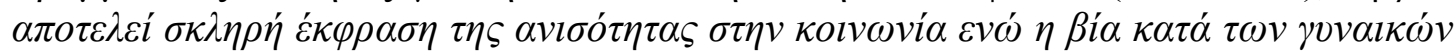

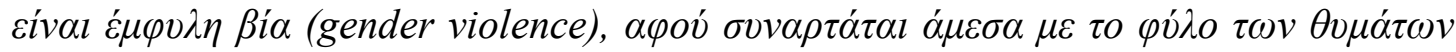
$\kappa \alpha l \tau \omega v \delta \rho \alpha \sigma \tau \omega ́ v »)$ 


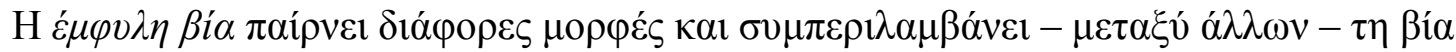

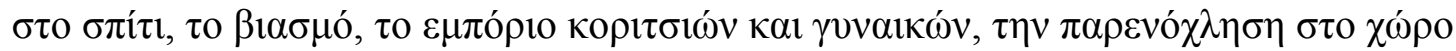

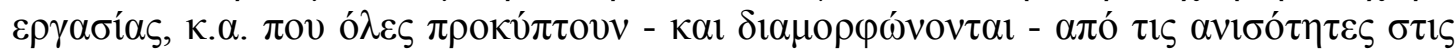

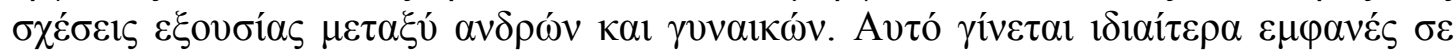

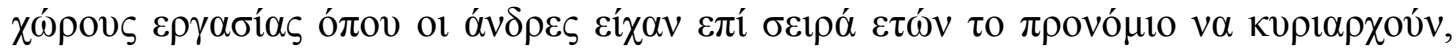

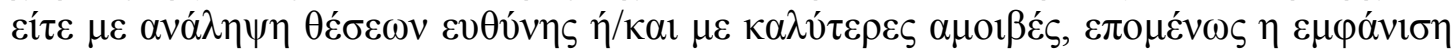

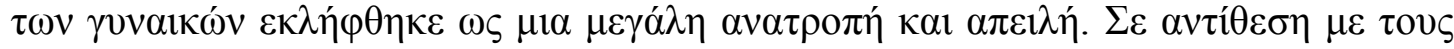

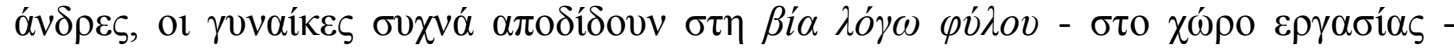

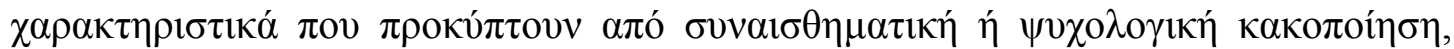

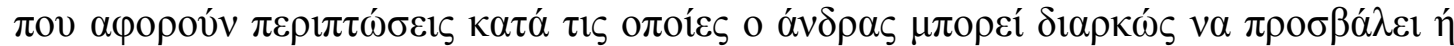

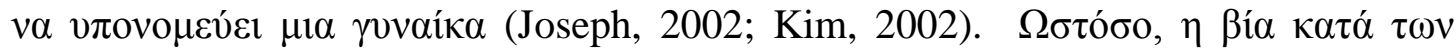

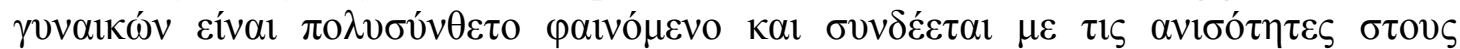

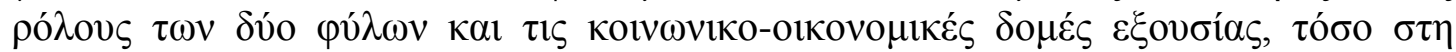

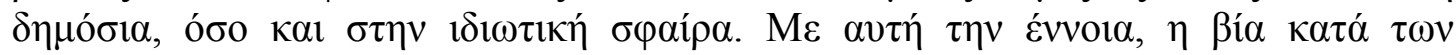

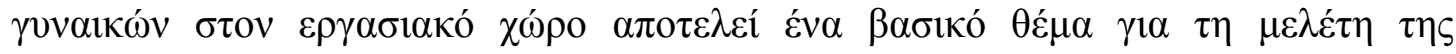

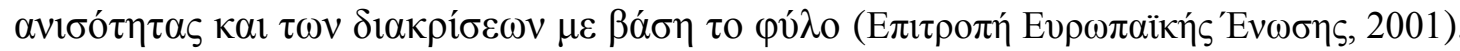

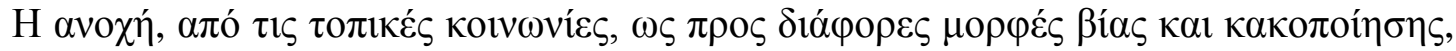

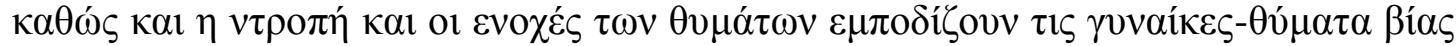

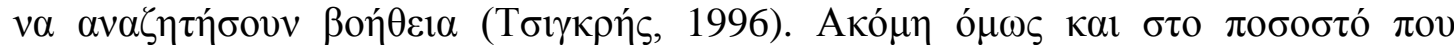

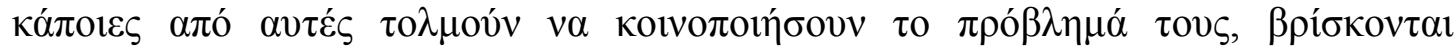

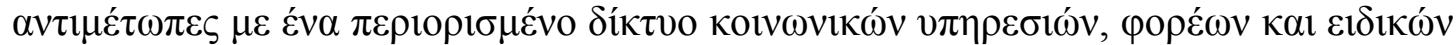

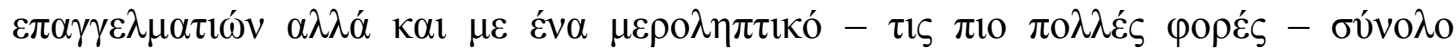

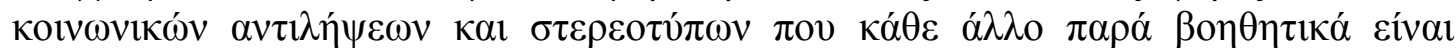

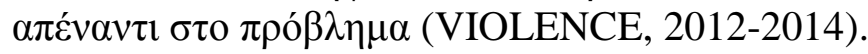

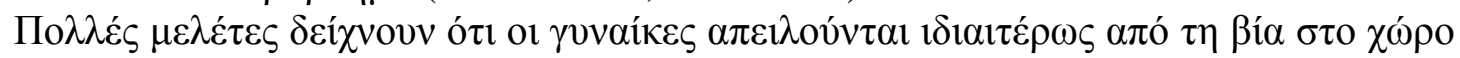

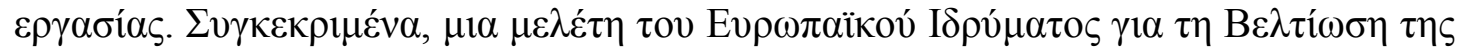
Z

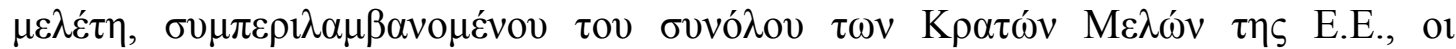

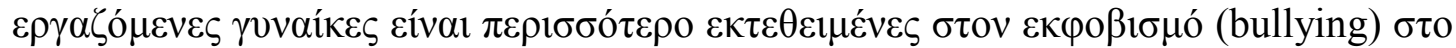

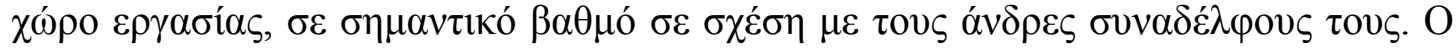

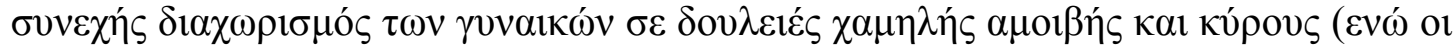

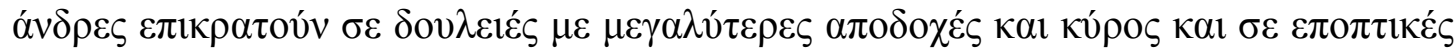

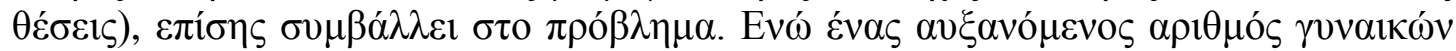

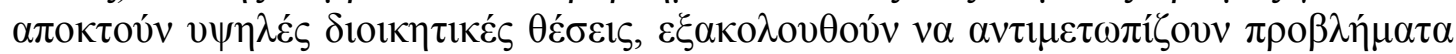

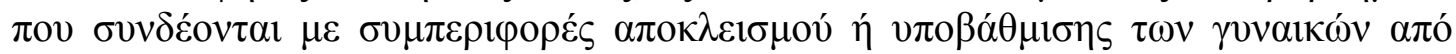

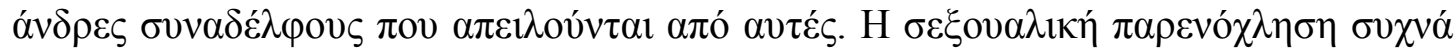

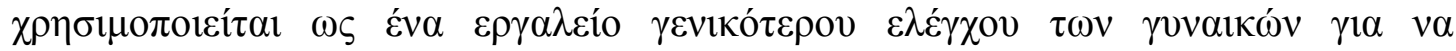

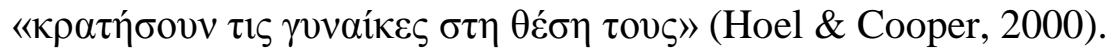

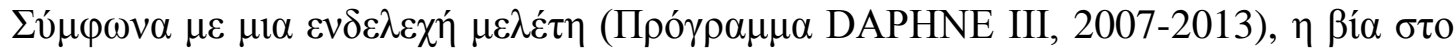

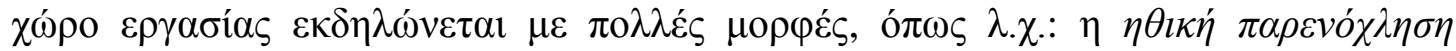

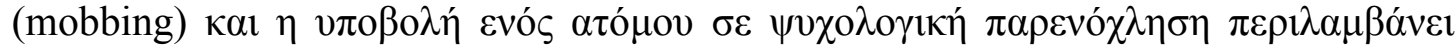

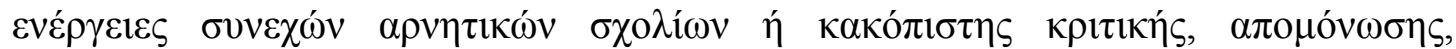

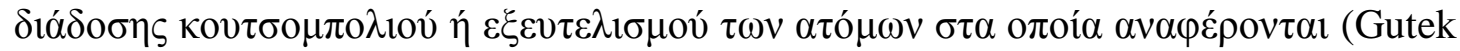

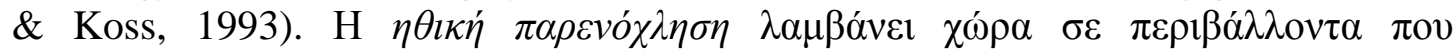

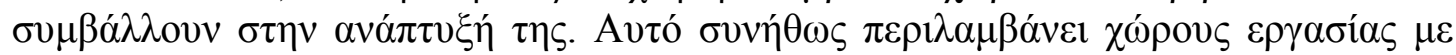

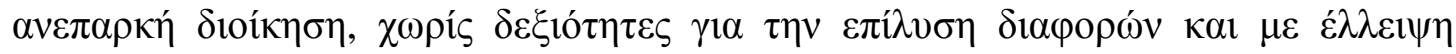

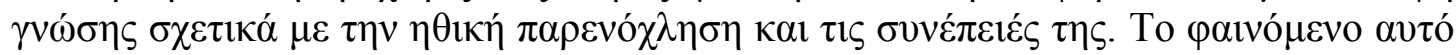

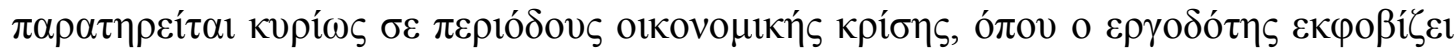
$\tau o v / \tau \eta v \varepsilon \varepsilon \gamma \alpha \zeta o ́ \mu \varepsilon v o-\eta$,

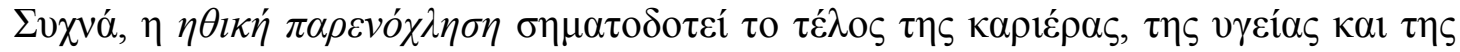




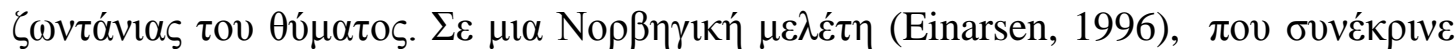

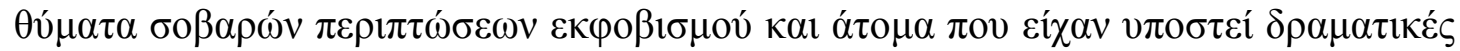

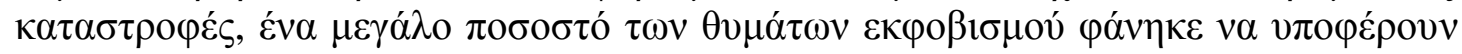
$\alpha \pi$ ó $\sigma v \mu \pi \tau \omega ́ \mu \alpha \tau \alpha \mu \varepsilon \tau \alpha \tau \rho \alpha v \mu \alpha \tau$

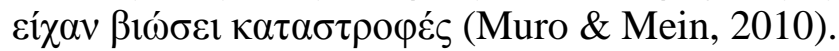

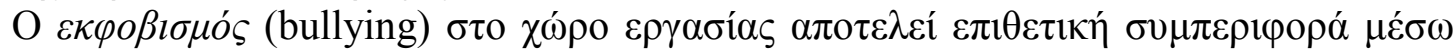

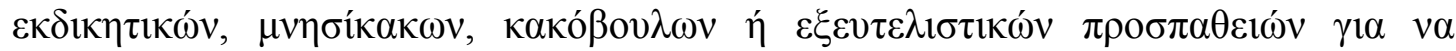

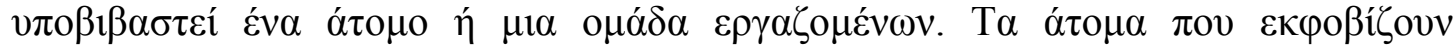

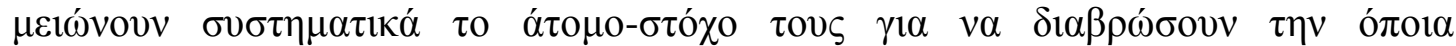

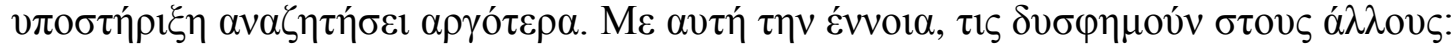

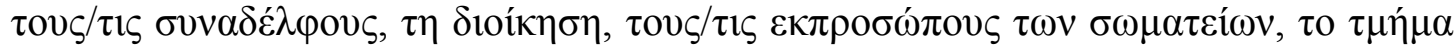

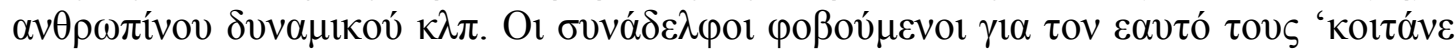

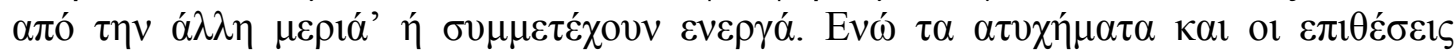

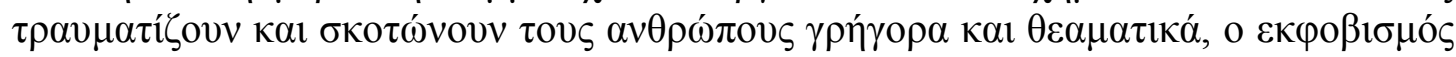

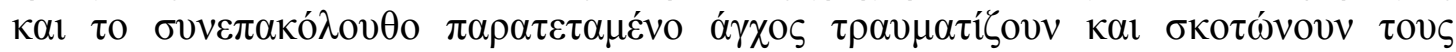

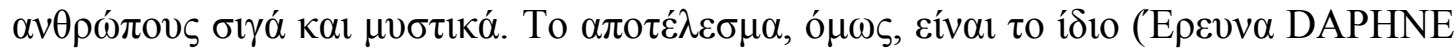
III, 2007-2013).

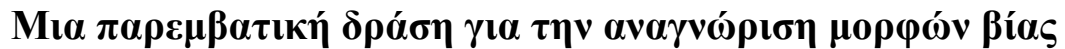

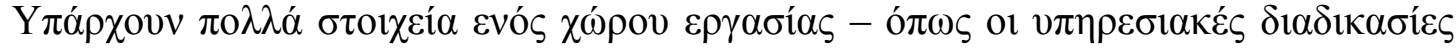

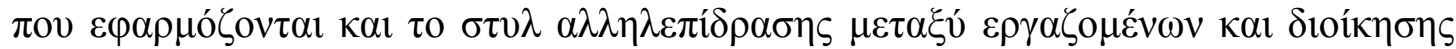

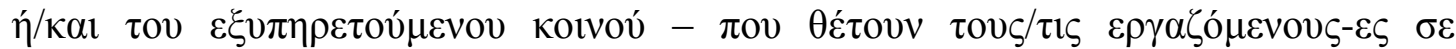

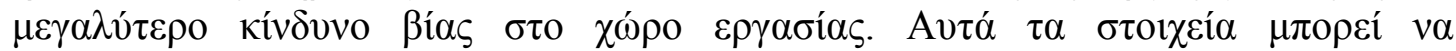

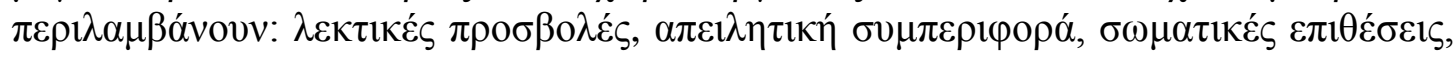

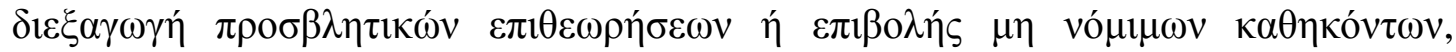

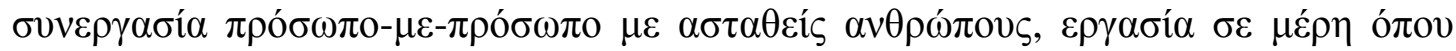

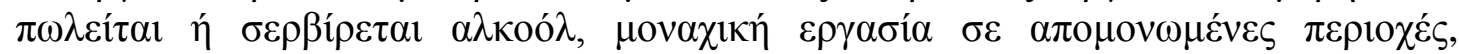

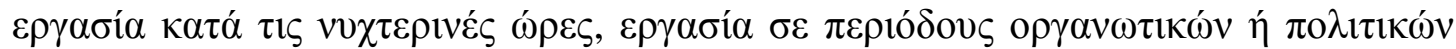

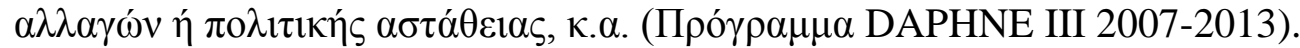

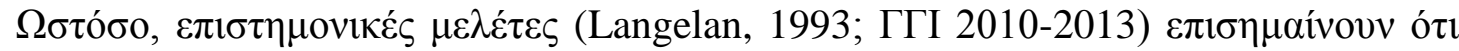

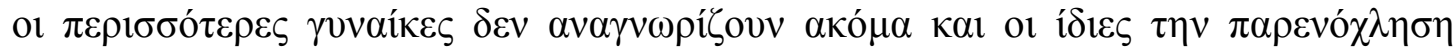

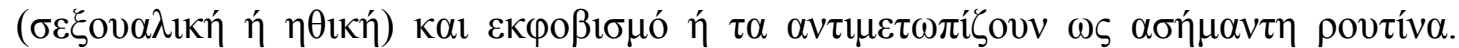

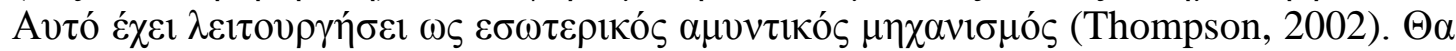

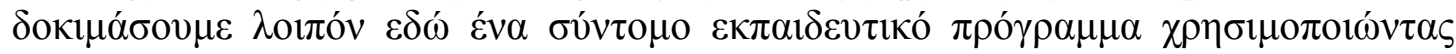

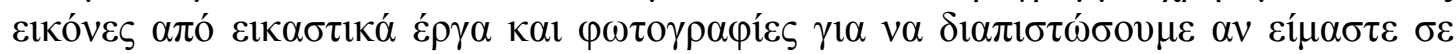

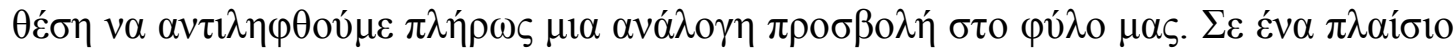

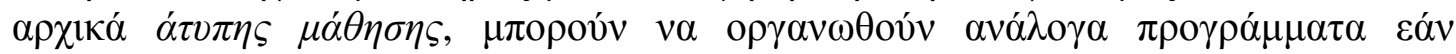

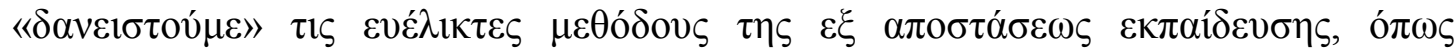

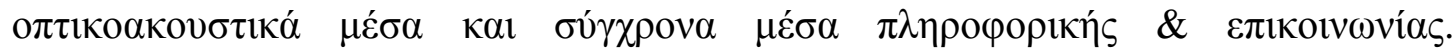

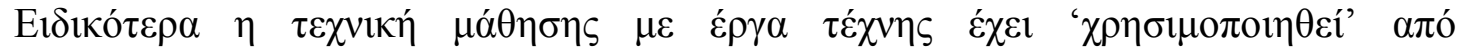

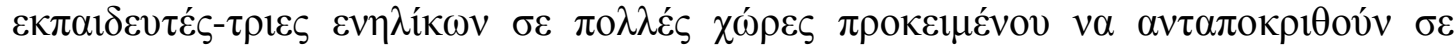

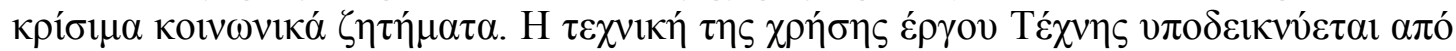

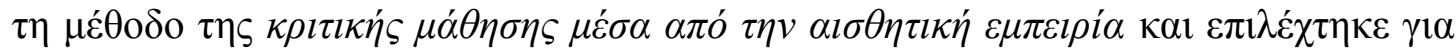

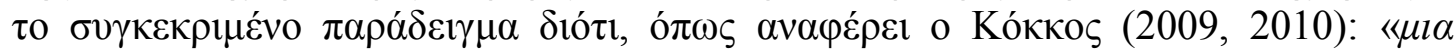

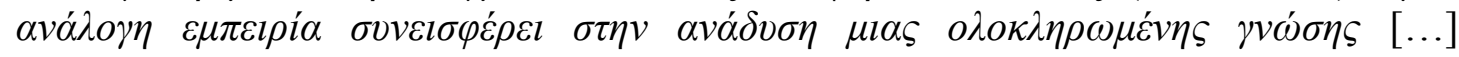

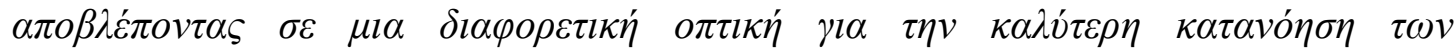

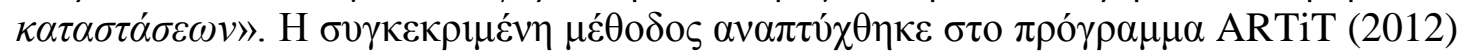

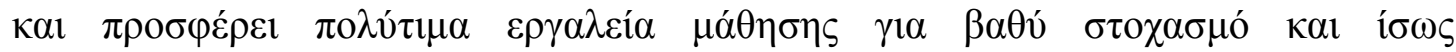

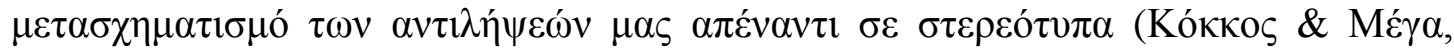

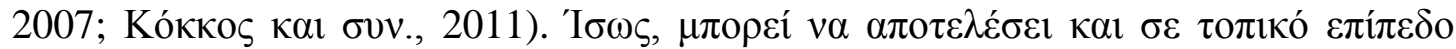




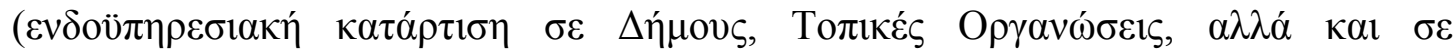

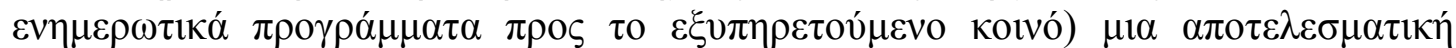

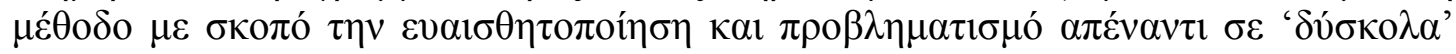

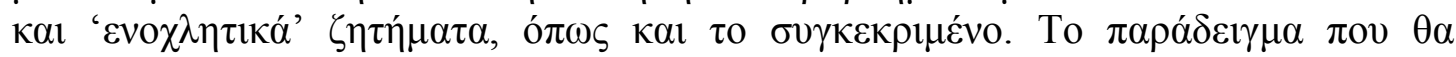

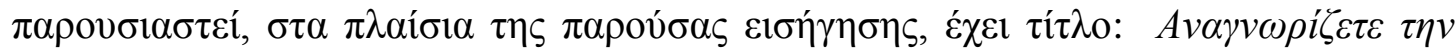
$\pi \alpha \rho \varepsilon v o ́ \chi \lambda \eta \sigma \eta$;

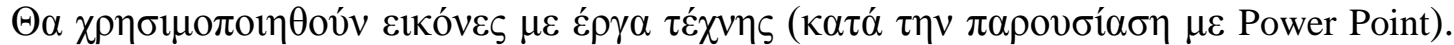

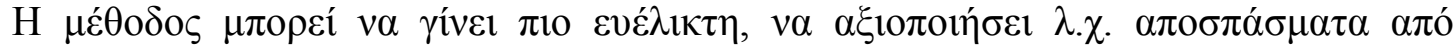

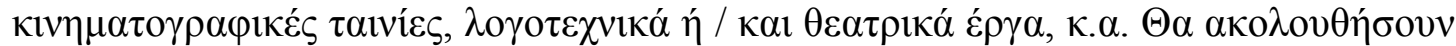

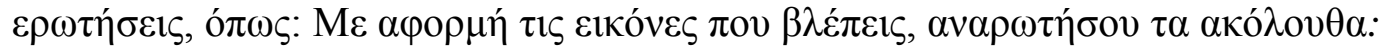

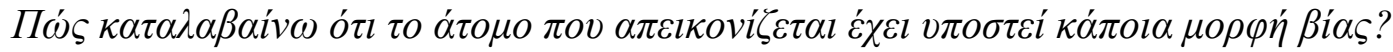

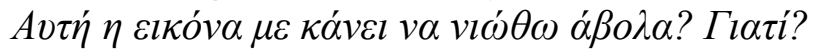

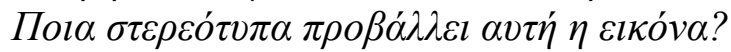

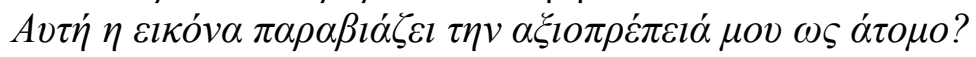

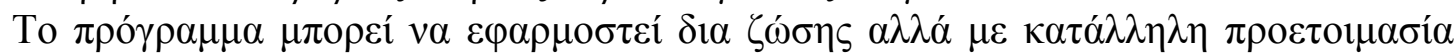

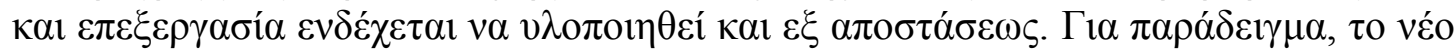

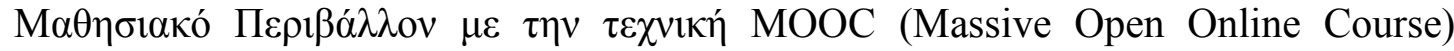

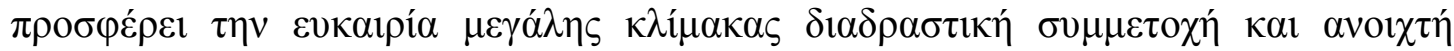

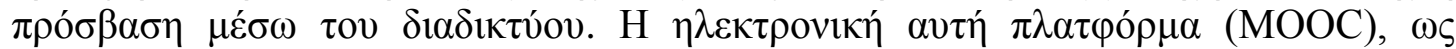

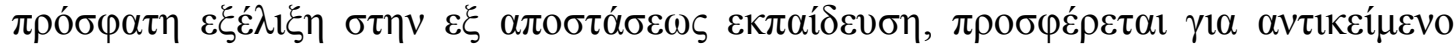

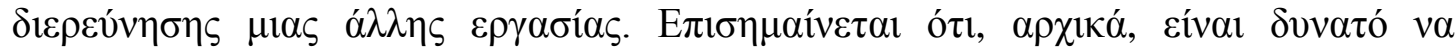

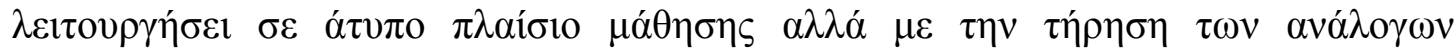

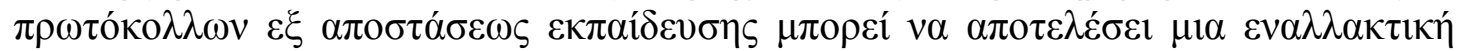

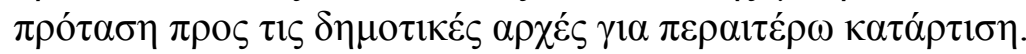

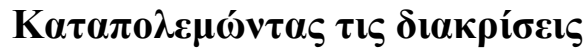

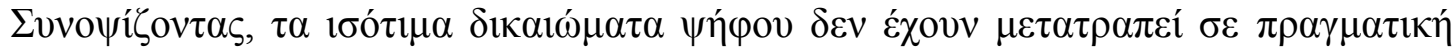

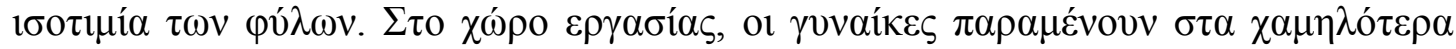

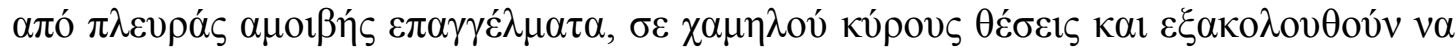

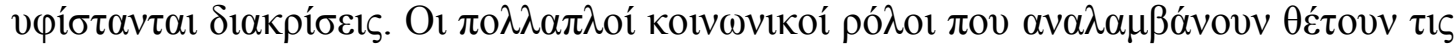

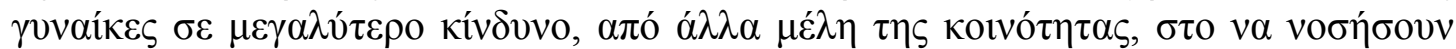

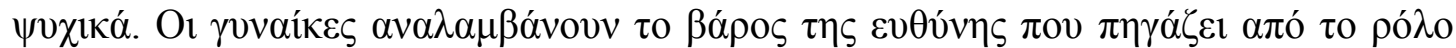

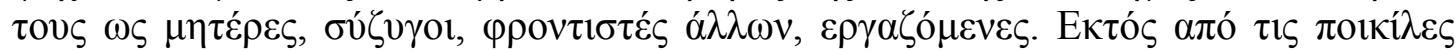

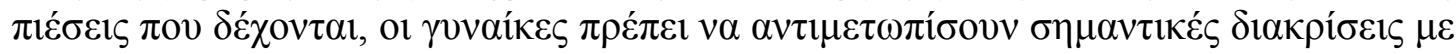

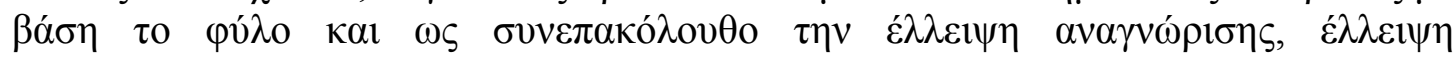

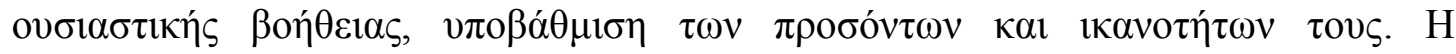

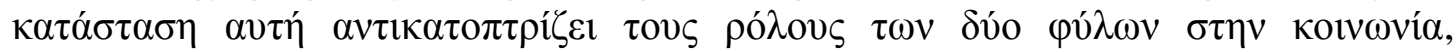

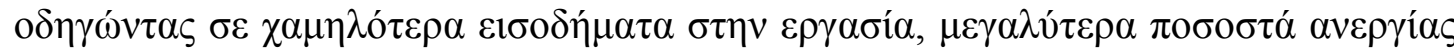

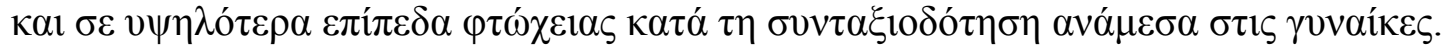

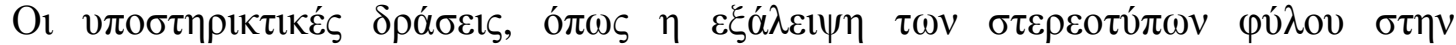

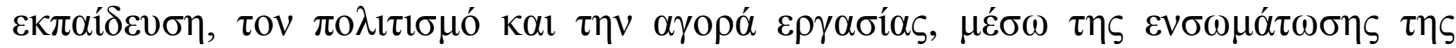

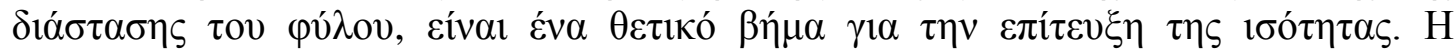

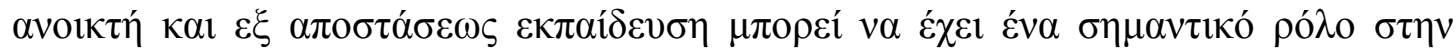

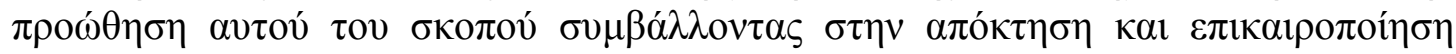

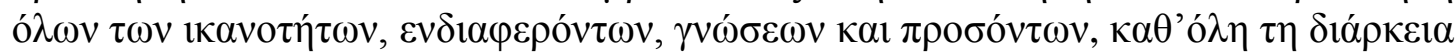

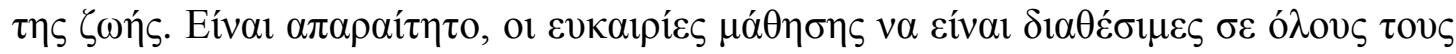

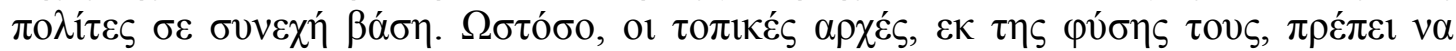

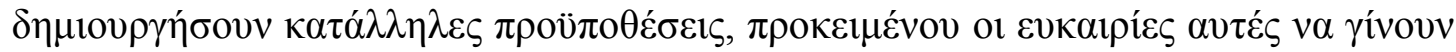

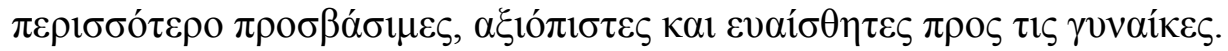




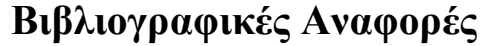

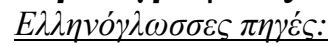

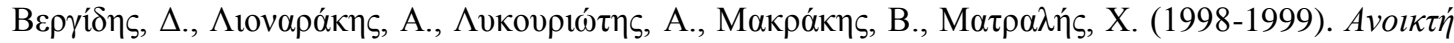

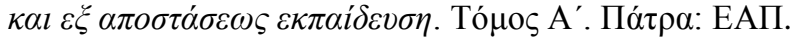

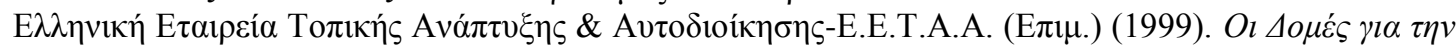

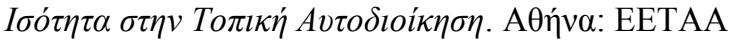

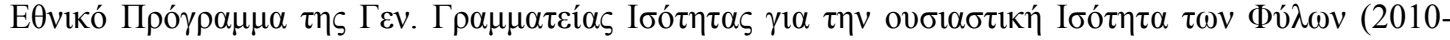

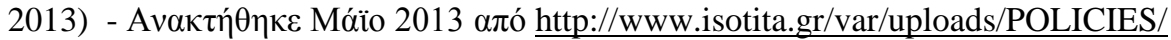

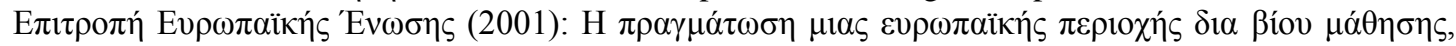

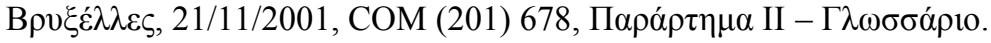

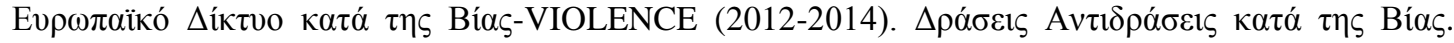

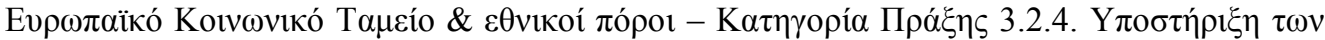

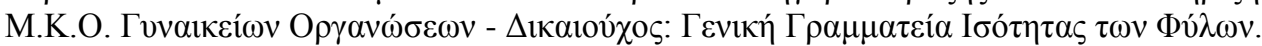

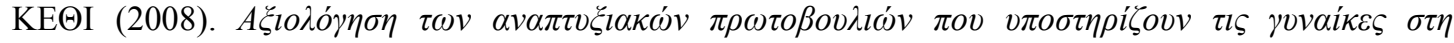

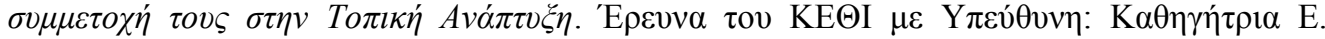

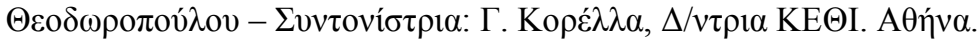

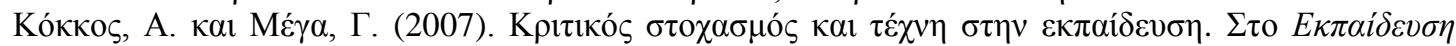

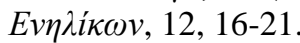

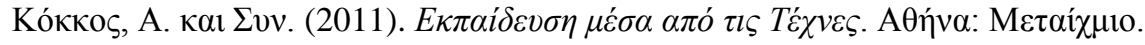

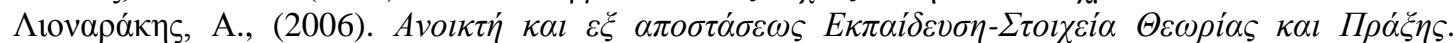

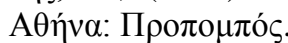

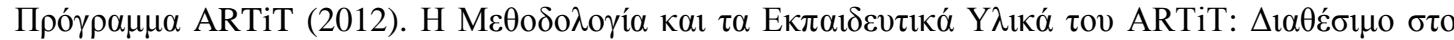
$\delta 1 \alpha \delta i ́ \kappa \tau v o$, http://artit.eu/gr/ARTiT\%20METHODOLOGY\%20GR.pdf

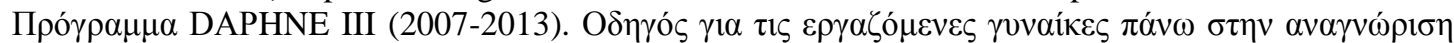

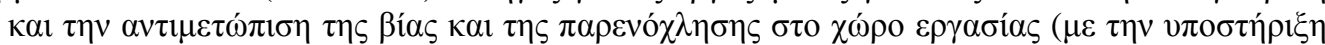

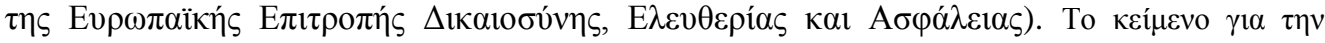

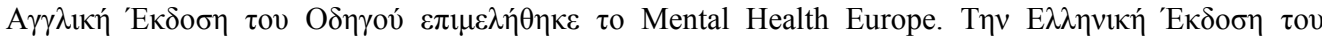

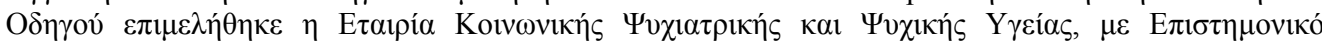

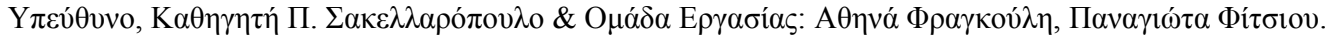

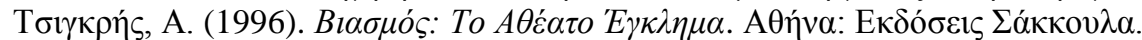

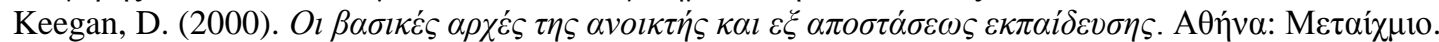

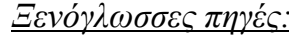

Barbieri, D, Bittarelli,B, Pesce, F, Bugeja, S, Spiteri, T, Agus, S, Polverari, L, Michie, R. (2007). Female Participation within Local Development. Programme concerning the European Community framework strategy on gender equality. Glasgow, UK: European Policies Research Centre-University of Strathclyde.

CEDAW (2010). The United Nations (UN) Entity for Gender Equality and the Empowerment of Women, July 2010. Retrieved from http://www.un.org/womenwatch/daw/cedaw/history.htm

European Foundation for the Improvement of Living and Working Conditions. (2007). 'Women and Violence at Work'. Retrieved from http://www.eurofound.europa.eu/pubdocs/2007/110/en/1/ef07110en.pdf

Gutek, B.A. \& Koss, M.P. (1993) Changed women and changed organisations: Consequences of and coping with sexual harassment. Journal of Vocational Behaviour, 42, 28-48.

Harding, S. (2003). The Feminist Standpoint Theory Reader: Intellectual and Political Controversies. London: Routledge.

Hoel, H. \& Cooper, C.L. (2000). Destructive Conflict and Bullying at Work. Unpublished Report. UK: UMIST.

Joseph, C. (2002). Gender and Local Government. SA: Friedrich Ebert Stiftung

Kim, J. \& Motsei, M. (2002). "Women enjoy punishment": attitudes and experiences of gender-based violence. In Social Science \& Medicine, 54, 1243-1254

Kokkos, A. (2009). 'Transformative Learning through Aesthetic Experience'. Paper presented at the 8th International Transformative Learning Conference - Bermuda. Available online: http://transformativelearning.org/index/TLC2009\%20Proceedings.pdf

Kokkos, A. (2010). Transformative Learning through Aesthetic Experience: Towards a Comprehensive Method. In Journal of Transformative Education, 8, 153-177.

Langelan, M. (1993). Back Off! How to Confront and Stop Sexual Harassment and Harassers. NY: Simon \& Schuster.

Merrill, B. (2005). 'Dialogical feminism: other women and the challenge of adult education'. in International Journal of Lifelong Education, 24, (1), 41-52. 
Muro, A. \& Mein, E. (2010). Domestic Trauma and Adult Education. in Adult Basic Education and Literacy Journal, 4(3), Fall, 2010: 140-150.

National Programme for Substantive Gender Equality (2010). Our Goal Substantive Gender Equality. Athens: KETHI - Retrieved June 2013 from www.kethi.gr

Seaforth, W. \& Mwaniki, E. (2008). Gender mainstreaming in local authorities: best practices. UN: United Nations Human Settlements Programme.

Tett, L. (2002). Community Education, Lifelong Learning, and Social Inclusion. Edinburgh, Scotland: Dunedin Academic Press.

Thompson, J. (2000). Women, Class and Education. London: Routledge.

Tisdell, E. (1998). 'Postructural feminist pedagogies: the possibilities and limitations of feminist emancipatory adult learning theory and practice'. in Adult Education Quarterly, Vol. 48, (3), 139-156.

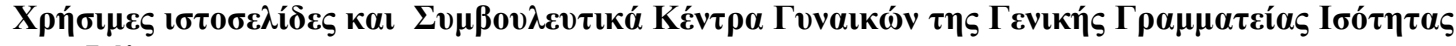

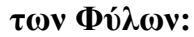

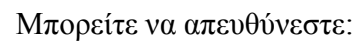 \\ - $\quad$ www.antiviolence-net.eu/FAQ.htm

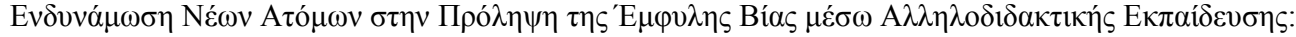 \\ Youth4Youth

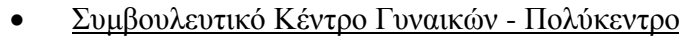

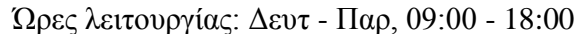

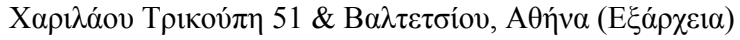

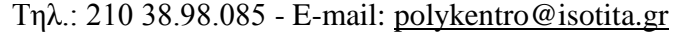

- $\quad \underline{\Sigma v \mu \beta 0 v \lambda \varepsilon v \tau \imath \kappa o ́ ~ K \varepsilon ́ v \tau \rho o ~ Г v v \alpha ı к \omega ́ v ~ A \theta \eta ́ v \alpha s ~}$

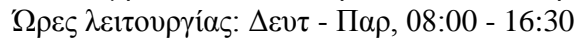

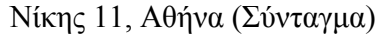

$\operatorname{T\eta } \lambda .: 210$ 33.17.305-6, E-mail: isotita4@otenet.gr

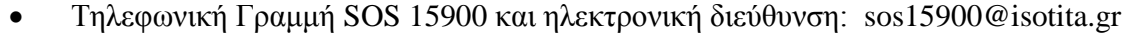

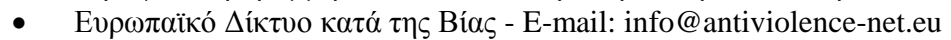

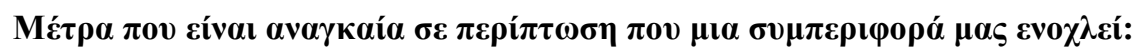

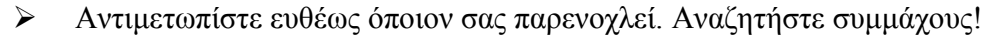

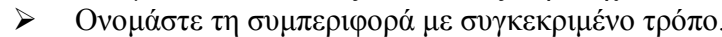

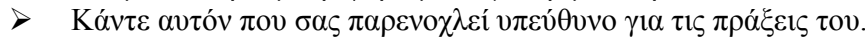

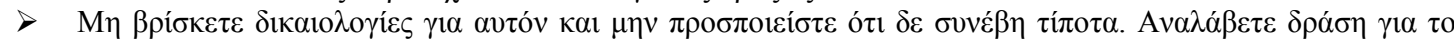

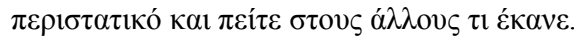

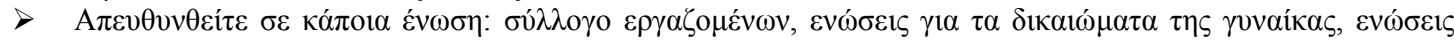

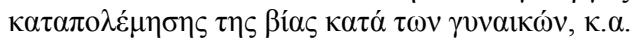

